\title{
A Formulation of Artificial Kansei Systems Based on Multi-agent Spaces Generated by Variable Neighborhood Models
}

\author{
Seiki UBUKATA*, Yasuo KUDO** and Tetsuya MURAI* \\ * Graduate School of Information Science and Technology, Hokkaido University, \\ Kita 14, Nishi 9, Kita-Ku, Sapporo, Hokkaido 060-0814, Japan \\ ** Muroran Institute of Technology, 27-1 Mizumoto-cho, Muroran, Hokkaido 050-8585, Japan
}

\begin{abstract}
In this paper, we formulate a method of designing artificial Kansei system based on a variable neighborhood model. We use a multi-agent space in order to realize a non-verbal system which can respond to individual situations. We carried out some simulation experiments of the system in which the system generates and produces some color arrangement like web pages as Kansei products. We also conducted a questionnaire survey in order to confirm that the system generates Kansei products which properly reflect users' Kansei.
\end{abstract}

Keywords: Artificial Kansei Designing, Variable Neighborhood Models, Color Combination, Color Image Scale

\section{INTRODUCTION}

In the field of Kansei engineering, Shiizuka [3] introduced a framework of Kansei system and proposed guiding principles for researches of artificial Kansei. In the framework, it is said to be the most important thing that a system can properly respond to individual situations.

Traditional Kansei systems treat measured Kansei data by statistical procedures. Because of this, individual Kansei come together as group Kansei. Hence, traditional Kansei systems cannot respond to individuals. In addition, the Kansei data which the system treats are measured at some point in the past. Hence, traditional Kansei systems cannot respond to current situation. In order to realize systems which respond to individual situations, system users need to interpose in processes of Kansei expression of the system in real time.

Traditional approaches to measure human Kansei are generally verbal approaches which use Kansei words. Transforming human Kansei to a finite set of Kansei words causes losses of human Kansei. Hence, artificial Kansei systems need Non-verbal approaches.

In this paper, we formulate a method of designing artificial Kansei systems based on variable neighborhood models [6] in order to realize a non-verbal system which responds to individual situations. The basic idea of our research is that we put our Kansei into multi-agent space and reflect our Kansei to Kansei expressions of the system.
We use a multi-agent space in which agents have neighborhoods (territories).

We carried out some simulation experiments of the system in which the system generates and produces some color arrangement like web pages as Kansei products. We use the Color Image Scale [4] to generate Kansei product from Kansei words. We also conducted a questionnaire survey in order to confirm that the system generates Kansei products which properly reflect users' Kansei.

\section{PRELIMINARIES}

In this section, we introduce a variable neighborhood model in order to realize non-verbal systems which have the capability to respond to each individual and each situation.

\subsection{Variable Neighborhood Model}

Ubukata et al. [5-7] proposed a variable neighborhood model in which autonomous agents change their sizes of neighborhoods. Variable neighborhood models are kinds of multi-agent systems. In a variable neighborhood model, agents exist in a topological space. Agents' ranges of views are taken as neighborhoods. Also agents' personal space [1] are taken as neighborhoods (Figure 1). Personal space is the region surrounding a person which they regard as psychologically theirs and if someone enters the space, he feels uncomfortable. Personal space is correlated well with human Kansei and personality. 


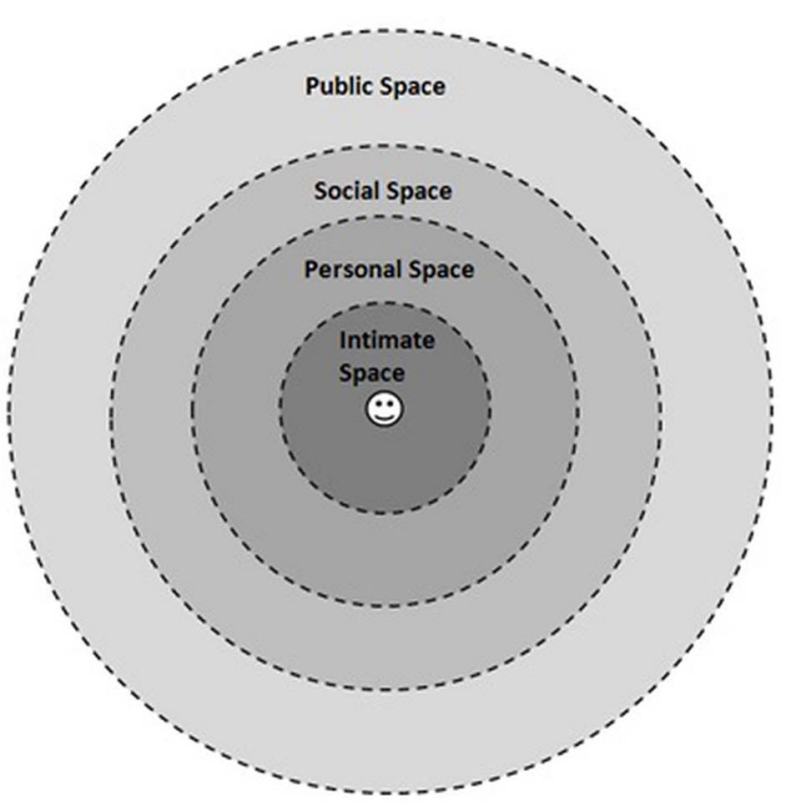

Figure 1: Personal space

A topological space is a pair $F=\langle\mathrm{U}, \mathrm{N}\rangle$, where $\mathrm{U}$ is a universe and $\mathrm{N}: \mathrm{U} \rightarrow \mathrm{P}(\mathrm{P}(\mathrm{U}))$ is a neighborhood system. Let $\mathrm{A}$ be a set of agents and $\mathrm{T}$ be a set of times. The location of an agent $i \in A$ at time $t \in T$ is written as $x_{i, t} \in U$. At time $t$, the agent $i$ settle a sequence of neighborhood

$$
\mathrm{U}_{\Lambda}^{\mathrm{i}, \mathrm{t}}=\left\langle\mathrm{N}_{1}\left(\mathrm{x}_{\mathrm{i}, \mathrm{t}}\right), \mathrm{N}_{2}\left(\mathrm{x}_{\mathrm{i}, \mathrm{t}}\right), \ldots, \mathrm{N}_{\Lambda}\left(\mathrm{x}_{\mathrm{i}, \mathrm{t}}\right)\right\rangle
$$

as range of view and personal space.

Ubukata et al. [7,8] discovered the fact that agents which have variable neighborhoods $U_{\Lambda}$ i,t behave as if they have some human Kansei through simulation experiments. For example, agents which tend to select small neighborhoods behave aggressively, offensively and bullishly. Conversely, agents which tend to select large neighborhoods behave passively, defensively and bearishly (Figure 2). The fact that these simple operations produce some Kansei is considered to be valuable in fundamental Kansei analysis or Kansei designing.

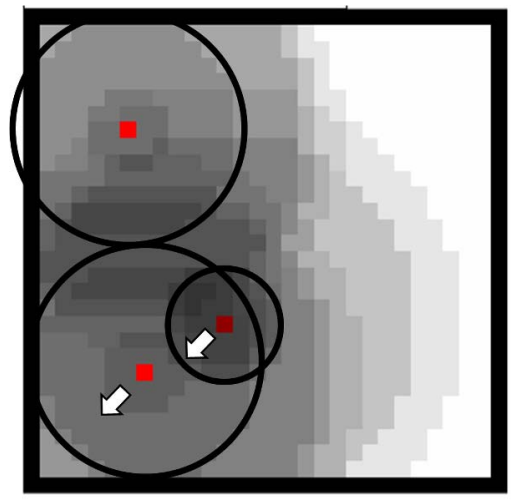

Figure 2: Invading behavior of an agent which shrinks his neighborhood in a multi-agent simulation

\section{ARTIFICIAL KANSEI SYSTEM DESIGNING BASED ON VARIABLE NEIGHBORHOOD MODELS}

\subsection{Ames of The System and Ideas for Realization}

In this section, we formulate a method of designing artificial Kansei system based on variable neighborhood models. We deal with a system which provides some Kansei products responding to individuals and current situations.

A most important thing for designing artificial Kansei system is that a system can properly respond to individual situations. In order to realize a system which responds to individual situations, system users need to interpose in processes of Kansei expression of the system in real time. In our approach, to realize this, system users act on a multi-agent space and agents in the space. In this section, we explain how to formulate designing artificial Kansei system based on a multi-agent space. As a first step, we design the system by verbal approach. In later step, we try to non-verbalize the system by reducing the verbal process.

\subsubsection{Basic Setting and Processing Flow}

We assume agents which have motion parameters which affect agents' behaviors. For instance we suppose a value of length of stride or a value of neighborhood size (territory size), etc. Let $\mathrm{P}$ be a set of agents motion parameters:

$$
\mathrm{P}=\mathrm{P}_{1} \times \mathrm{P}_{2} \times \ldots \times \mathrm{P}_{\mathrm{N}} .
$$

For instance agents have parameters:

Length of Stride: $\mathrm{P}_{1}=[1,5]$,

Neighborhood Size: $P_{2}=[10,100]$ :

System users can operate these parameters in real time and affect agents' behaviors. Real-time individual operations of these parameters make it possible that the system responds to individual situations. These operations make it possible to generate behaviors which match users' current moods. Let $\mathrm{B}_{\mathrm{A}}$ be a set of agents' behaviors. Designing the mechanism which reflects these parameters in agents' behaviors is comparable to make the correspondence

$$
\mathrm{G}_{\mathrm{P}-\mathrm{BA}}: \mathrm{P} \rightarrow \mathrm{B}_{\mathrm{A}} .
$$

$\mathrm{BA}$ is not determined uniquely because of randomness and external factor. Let KW be a set of Kansei words. Below is an example of a set of Kansei words:

$$
\mathrm{KW}=\{\text { "bold", "active", "wild", "mild", ... }\} \text {. }
$$


The system extracts some Kansei words from current agents' behaviors and generates some Kansei product based on the Kansei words. We need to measure human Kansei which appear in users mind from agents' behaviors by questionnaires previously. That is, we prepare a correspondence

$$
\mathrm{G}_{\mathrm{BA}-\mathrm{KW}}: \mathrm{B}_{\mathrm{A}} \rightarrow \mathrm{P}(\mathrm{KW})
$$

in advance. By this measurement, we can obtain the mapping from motion parameters to Kansei words:

$$
f_{\mathrm{P}-\mathrm{KW}}: \mathrm{P} \rightarrow \mathrm{P}(\mathrm{KW})
$$

using correspondences $\mathrm{G}_{\mathrm{P}-\mathrm{BA}}$ and $\mathrm{G}_{\mathrm{BA}-\mathrm{KW}}$. At this process, suitable treatment which converts correspondences into the mapping is needed. Let KP be a set of Kansei products. There are traditional methods of Kansei system which generate Kansei products based on given Kansei words. Designing such systems is comparable to make the mapping

$$
f_{\mathrm{KW}-\mathrm{KP}}: \mathrm{P}(\mathrm{KW}) \rightarrow \mathrm{P}(\mathrm{KP}) .
$$

Using the correspondence $\mathrm{G}_{\mathrm{P}-\mathrm{BA}}, \mathrm{G}_{\mathrm{BA}-\mathrm{KW}}$ and the mapping $f_{\mathrm{P}-\mathrm{KP}}$, we can obtain the mapping

$$
f_{\mathrm{P}-\mathrm{KP}}: \mathrm{P} \rightarrow \mathrm{P}(\mathrm{KP}) .
$$

Transformation image:

$$
\mathrm{P} \rightarrow \mathrm{B}_{\mathrm{A}} \rightarrow \mathrm{P}(\mathrm{KW}) \rightarrow \mathrm{P}(\mathrm{KP})
$$

Users operate the parameters based on current mood and get a generated Kansei product. Users view both agents' behaviors and Kansei products, operating the parameters.

$$
\begin{gathered}
f_{\mathrm{P}-\mathrm{KP}}: \mathrm{P} \rightarrow \mathrm{P}(\mathrm{KP}), \\
f_{\mathrm{P}-\mathrm{BA}}: \mathrm{P} \rightarrow \mathrm{B}_{\mathrm{A}} .
\end{gathered}
$$

Figure 3 shows the basic processing flow of this system.

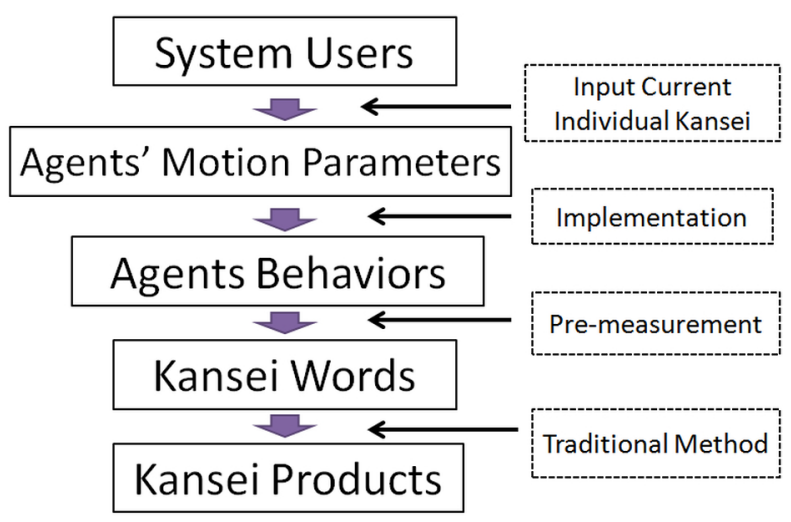

Figure 3: Basic Processing Flow

\subsection{Measurement Processes of Kansei}

This system uses a mapping from agents' behaviors to Kansei words. In order to implement the system, the mapping needs to be measured preliminarily. If some parameters have a number of values, like real-valued parameters, it is difficult to obtain the mapping. So, we need to granulate the space $\mathrm{P}$ of parameters into P'. For example, we divide each parameter into two intervals. "S" means the value is small and "L" means the value is large.

$$
\begin{aligned}
& \mathrm{P}=\mathrm{P} 1^{\prime} \times \mathrm{P} 2^{\prime} \times \mathrm{P} 3^{\prime}, \\
& \mathrm{P} 1{ }^{\prime}=\{[10,50),[50,100]\}=\{“ \mathrm{~S} ”, " \mathrm{~L} ”\} \text {, } \\
& \mathrm{P} 2{ }^{\prime}=\{[1,3),[3,5]\}=\{“ \mathrm{~S} ", \text { "L” }\}, \\
& \mathrm{P} 3{ }^{\prime}=\{[0,0: 5),[0: 5,1]\}=\left\{{ }^{\prime S} ", " L "\right\},
\end{aligned}
$$

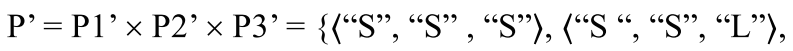

$$
\begin{aligned}
& \ldots,\langle\text { "L", "L", "L “ " }\rangle\} \text {. }
\end{aligned}
$$

By this granulation, combinations of parameters become 8 patterns. We observe agents' behaviors generated based on these combinations and measure Kansei words which come to our mind. Using obtained mapping, we can get some Kansei words from a combination of parameters.

For instance, if a user selects a combination 〈"S", "S", "L" $\rangle$ then Kansei words "dynamic" and "wild" are obtained by the mapping

$$
f(\langle\text { 'S", "S", "L”) })=\{\text { “dynamic", "wild" }\} \text {. }
$$

System users can put their Kansei into the multi-agent space by operating various parameters with viewing agents' behavior.

\subsection{Expression Processes of Kansei}

There are many previous works which generate Kansei products from Kansei words. For example, Inoue [2] use the color image scale [4] to generate Kansei products (uniform color combination). By learning from [2], we leverage the Color Image Scale in the expression processes of the system.

\subsubsection{The Color Image Scale}

Kobayashi [4] developed Color Image Scale [9] (Figure 4). Kobayashi and his team at the Nippon Color \& Design Research Institute have matched 130 basic colors and over 1,000 color combinations to 180 key image words (Kansei words). If we want to make an interior or an outfit "elegant" or "romantic", we look up the word in the key image word index, and choose any one of the dozens of color combinations listed under the entry. Conversely, if we would like to know what mood certain colors or color combinations suggest, refer to the color index. Used by thousands of major designers and manufacturers in Japan, 


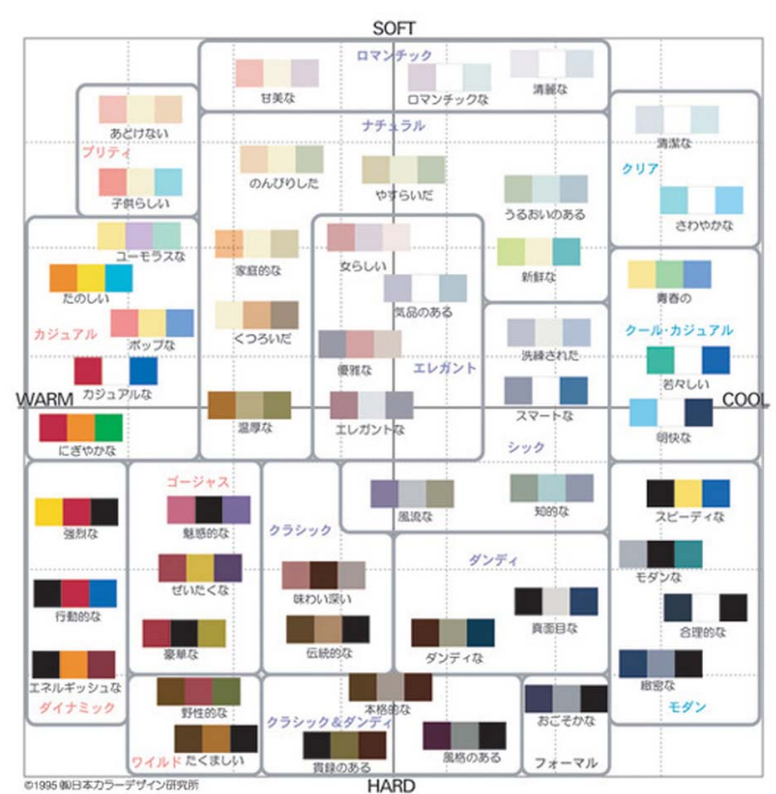

Figure 4: The Color Image Scale [9]

the Color Image Scale is a unique color resource for both professionals and amateurs alike.

\subsection{Tuning of the System}

In case of a system user make a request to agents to do "dynamic" behaviors, the system should output Kansei products which give the user a "dynamic" impression. However, agents' behaviors make a transformation into Kansei products which have different structure. So, we may feel different impression from these. In order to reduce the gap, we need to determine the gap between Kansei words from agents' behaviors and Kansei words from Kansei products and give some feedback to the system. Two correspondences

$$
\begin{aligned}
& \mathrm{G}_{\mathrm{BA}-\mathrm{KW}}: \mathrm{B}_{\mathrm{A}} \rightarrow \mathrm{P}(\mathrm{KW}), \\
& \mathrm{G}_{\mathrm{KP}-\mathrm{KW}}: \mathrm{KP} \rightarrow \mathrm{P}(\mathrm{KW})
\end{aligned}
$$

should be compared.

\subsection{Non-verbalization of The System}

In a traditional approach, users need to input or select some Kansei words and give it to the system. Our new system uses Kansei words in internal processing. So, our system is based on verbal approach. However, in this system, users do not use Kansei words directly. Users make physical operation of the parameters through the user interface of the system and get Kansei products. This is non-verbal interactions. At present, this is superficially non-verbal system. We think this type of multi-agent space has possibilities of realizing completely non-verbal method. We think that an approach using the whole body is expected.

\section{SIMULATION EXPERIMENTS}

We carried out some simulation experiments using the proposed method (Figure 5). We make a system which generates and produces a layout like web pages which have color combinations as Kansei product.

Agents exist in a 2-dimensional Euclidean space which is one of topological spaces. This system consists of the following components:

1. A user interface which make it possible to operate some agents motion parameters

2. A region which displays agents' behaviors

3. A region which displays a generated image which have color combination

\subsection{The Implementation of The Method}

We set up the following agents' motion parameters:

$$
\begin{gathered}
\mathrm{P}=\mathrm{P}_{\mathrm{N}} \times \mathrm{P}_{\mathrm{S}} \times \mathrm{P}_{\mathrm{R}}, \\
\mathrm{P}_{\mathrm{N}}=[10,100], \mathrm{P}_{\mathrm{S}}=[1,5], \mathrm{P}_{\mathrm{R}}=[0,1] .
\end{gathered}
$$

Each parameter denotes the following characteristics.

1. Size of neighborhood: This value means a size of neighborhood of an agent. For simplicity, we assume that one agent have one neighborhood.

2. Strenuous: An agent's length of stride increase as this value increases.

3. Randomness: An agent turns for direction randomly as this value come nearer to 1 .

We granulate these parameters as below:

$$
\begin{gathered}
\mathrm{P}_{\mathrm{N}}{ }^{\prime}=\{[10,50),[50,100]\}=\{“ \mathrm{~S} ", " \mathrm{~L} "\}, \\
\mathrm{P}_{\mathrm{S}}{ }^{\prime}=\{[1,3),[3,5]\}=\{“ \mathrm{~S} ", " \mathrm{~L} "\}, \\
\mathrm{P}_{\mathrm{R}}{ }^{\prime}=\{[0,0.5),[0.5,1]\}=\{“ \mathrm{~S},, " \mathrm{~L} "\} .
\end{gathered}
$$

We measured some correspondence between agents' motion parameters and Kansei words which pop up in users mind from agents' behaviors. Table 1 shows the result of the measurement.

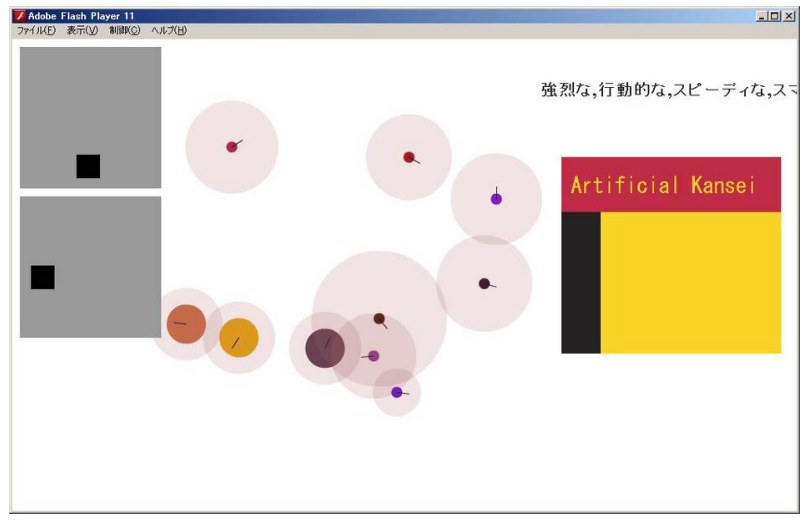

Figure 5: Multi agent simulation 
Table 1: The mapping $f_{\mathrm{P}-\mathrm{KW}}$ from the granulated parameter combinations to Kansei words

\begin{tabular}{|c|c|}
\hline Motion Parameters & Kansei Words \\
\hline$\langle " S ", " S ", " s "\rangle$ & $\{$ \{elegant" $\}$ \\
\hline$\langle " S ", " S ", " L "\rangle$ & $\{$ "pop" $\}$ \\
\hline$\langle " S ", " L ", " S "\rangle$ & \{"pop", "active”, "speedy", "smart”\} \\
\hline$\langle " S ", " L ", " L "\rangle$ & $\{$ “active", "wild"' $\}$ \\
\hline$\langle “ L ", " S ", " S "\rangle$ & \{“mild", "leisurely", "relaxing"\} \\
\hline$\langle " L ", " s ", " L "\rangle$ & $\{$ "young", "naïve" $\}$ \\
\hline$\langle " L ", " L ", " S "\rangle$ & \{"flowing" $\}$ \\
\hline$\langle$ "L", "L", "L"〉 & $\{$ "energetic" $\}$ \\
\hline
\end{tabular}

If a user operates parameters, the system obtains a set $\mathrm{W}$ of Kansei words from the mapping. The system selects a Kansei word $\mathrm{w} \in \mathrm{W}$ randomly and searches a color combination from the Color Image Scale and coordinates colors.

\subsection{Results of Simulation Experiments}

In this subsection, we present Kansei products actually obtained based on parameter combinations selected by a system user.

\subsection{1 〈“'S", "S", "S"〉}

A Kansei word "elegant" is obtained by the mapping $f_{\mathrm{P}-\mathrm{KW}}(\langle " \mathrm{~S} "$, "S", "S"〉) $=\{$ "elegant" $\}$. The system searches a color combination which is "elegant" from the Color Image Scale and arranges the product. The generated product is Table 2. Presentations of results are presented in the same way hereinafter.

\subsection{2 〈“S", "S", "L"〉}

The calculated result of the mapping is $f_{\mathrm{P}-\mathrm{KW}}(\langle$ " $\mathrm{S}$ ", " $\mathrm{S}$ ", "L”〉) $=\{$ “pop" $\}$.

\subsection{3〈“'S, "L", "S"〉}

The calculated result of the mapping is $f_{\mathrm{P}-\mathrm{KW}}(\langle$ "S", "L ", "S" $\rangle)=\{$ "bold", "active", "speedy", "smart" $\}$.

\subsection{4 〈'S", “L”, “L”〉}

The calculated result of the mapping is $f_{\mathrm{P}-\mathrm{KW}}(\langle<\mathrm{S}$, "L, "L $\rangle)=\{$ "active", "wild" $\}$

\subsection{5 〈'L", “S", "S"〉}

The calculated result of the mapping is $f_{\mathrm{P}-\mathrm{KW}}(\langle$ " $\mathrm{L}$ ", "S", "S“ $\rangle)=\{$ "mild", "leisurely", "relaxing" $\}$.
Table 2: Results (Kansei products)

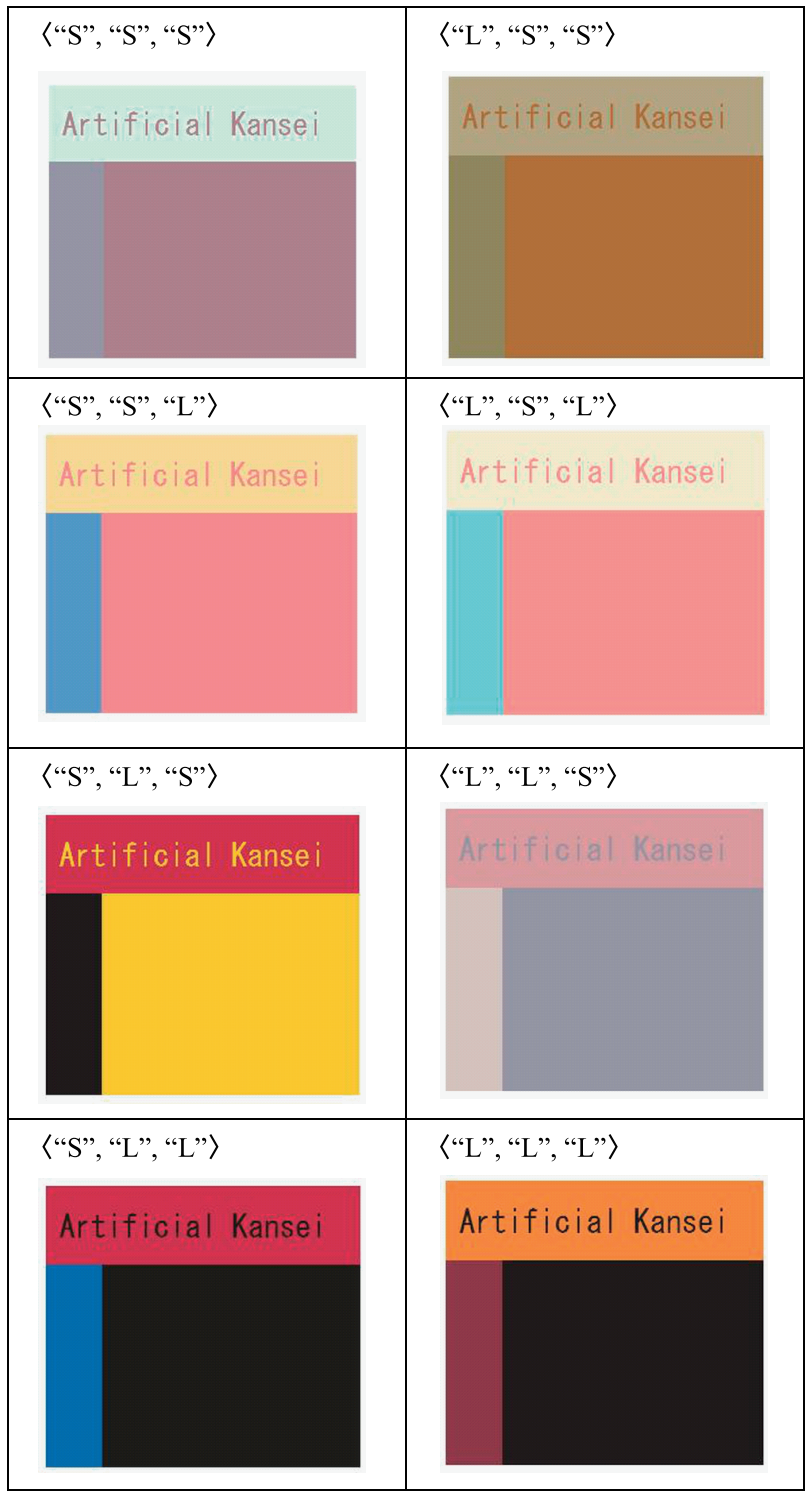

\subsection{6〈“'L", "S", “L”〉}

The calculated result of the mapping is $f_{\mathrm{P}-\mathrm{KW}}(\langle$ "L", " $\mathrm{S}$ ", "L" $\rangle)=\{$ "young", "naïve naive" $\}$.

\subsection{7 〈“L”, “L”, “S”〉}

The calculated result of the mapping is $f_{\mathrm{P}-\mathrm{KW}}(\langle$ ' $\mathrm{L} "$, " $\mathrm{L}$ ", "S" $\rangle)=\{$ "flowing" $\}$.

\subsection{8 〈“L", "L", "L"〉}

The calculated result of the mapping is $f_{\mathrm{P}-\mathrm{KW}}(\langle$ "L", " $\mathrm{L}$ ", "L") $=\{$ "energetic" $\}$.

\subsection{The Questionnaire Survey and The Results}

We conducted a questionnaire survey to students in order to confirm that the system generates Kansei products which properly reflect users' Kansei. In this subsection, we present the questionnaire result and a review of the 
results. In the questionnaire survey, we asked the degree of coincidence between Kansei, impression and moods from agents' behaviors and those from Kansei products (color combinations). Table 3 shows the questionnaire result. "-2" is "Very mismatch", "-1" is "Mismatch", "0" is "Neither", "1" is "Match" and " 2 " is "Very match".

According to the survey, we can infer the tendency to estimate that Kansei from agents' behaviors corresponds to Kansei from color combinations. However, for example, the result of the case $\langle$ "L", "L", "S"〉 is not good. In this case, designers of the system needs appropriate tuning. This result means that non-verbal approach in which users don't use Kansei word can reflect users Kansei.

When the user feels overwrought, he sympathizes with agents which behave strenuously, and loud and provocative color combinations are generated. And when the user feels comfortable, he sympathizes with agents which behave fluently, and subdued color combinations are generated. In this way the user's moods for agents' behaviors generate reasonably suitable Kansei products. By these results, we conclude that the system reflect user's current mood in the Kansei product appropriately.

\section{CONCLUDING REMARKS}

In this paper, we formulated a method of designing artificial Kansei system based on a variable neighborhood model. This system has a non-verbal aspect and a capability of responding to individual situations. We carried out some simulation of the proposed method and conducted a questionnaire survey and confirmed that the system reflects a user's current mode in the Kansei product

Table 3: The questionnaire result

\begin{tabular}{|c|c|c|c|c|c|}
\hline & -2 & -1 & 0 & 1 & 2 \\
\hline$\langle " S ", " S ", " S "\rangle$ & 0 & 1 & 1 & 4 & 0 \\
\hline$\langle “ S ", " S ", " L "\rangle$ & 0 & 1 & 2 & 2 & 1 \\
\hline$\langle “ S ", " L ", " S "\rangle$ & 0 & 0 & 2 & 4 & 0 \\
\hline$\langle “ S "$, "L”, "L”〉 & 1 & 0 & 2 & 1 & 2 \\
\hline$\langle " L ", " S ", " S "\rangle$ & 0 & 0 & 0 & 3 & 3 \\
\hline$\langle “ L ", " S ", " L "\rangle$ & 0 & 1 & 2 & 2 & 1 \\
\hline$\langle “ L ", " L ", " S "\rangle$ & 0 & 4 & 2 & 0 & 0 \\
\hline$\langle “ L ", " L ", " L "\rangle$ & 0 & 3 & 2 & 1 & 0 \\
\hline Total & 1 & 10 & 13 & 17 & 7 \\
\hline
\end{tabular}

appropriately on some level. Human Kansei is a concept which is intimately related to implicit knowledge and body intelligence. Hence, artificial Kansei systems in which agents work for users' hand and foot are considered to be well suited to Kansei realization. We plan to apply this approach in various sorts of Kansei designing and confirm its utility.

\section{ACKNOWLEDGEMENTS}

The authors would like to thank the anonymous reviewers for their valuable comments and suggestions to improve the quality of the paper.

\section{REFERENCES}

1. E. T. Hall; The Hidden Dimension, Doubleday, 1966.

2. H. Inoue, Y. Muramoto; Color Combination System Using Emotion Color Combination Model and Image Emotion Model and its Application to Uniform Color Combination, Transactions of Japan Society of Kansei Engineering, 11(2), pp.273-280, 2012.

3. H. Shiizuka; Overview of Kansei System and Related Problems, Second International Conference, RSKT 2007, Proceedings, pp.203-210, 2007.

4. S. Kobayashi; Color Image Scale, Kodansha USA, 1992.

5. S. Ubukata, Y. Kudo, and T. Murai; An Agent Control Method Based on Rough-set-based Granularity, Soft Computing and Intelligent Systems and 9th International Symposium on Advanced Intelligent Systems (SCIS \& ISIS 2008), CD-ROM, FR-G3-1, 2008.

6. S. Ubukata, Y. Kudo, and T. Murai; An Agent Control Method Based on Variable Neighborhoods, KnowledgeBased and Intelligent Information and Engineering Systems, LNAI 5712, Springer, Berlin, pp.356-363, 2009.

7. S. Ubukata, Y. Kudo, and T. Murai; Autonomous Agent Control Based on Variable Neighborhoods, International Journal of Reasoning-based Intelligent Systems (IJRIS), 3(1), 2010.

8. S. Ubukata, Y. Kudo, and T. Murai; Agents' Kansei Expression Based on Variable Neighborhood Models, The 2011 IEEE International Conference on Granular Computing (GrC2011), A4-05, 2011.

9. NIPPON COLOR \& DESIGN RESEARCH INSTITTE INC. (NCD); http://www.ncd-ri.co.jp 


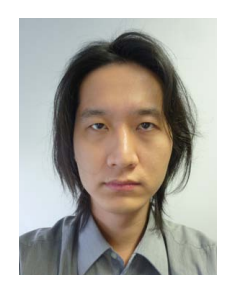

\section{Seiki UBUKATA}

Seiki Ubukata is a doctoral student at Graduate School of Information Science and Technology, Hokkaido University, Hokkaido, Japan. He received the degree of Bachelor of Engineering and Master of Information Science and Technology from Hokkaido University, in 2007 and 2009, respectively. His current research interests are theory and application of rough sets, modal logic, topological spaces, granular computing, affective engineering, Kansei engineering, and autonomous agent control.

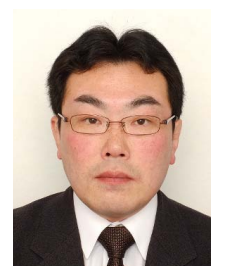

\section{Yasuo KUDO}

Yasuo Kudo is an Associate Professor at College of Information and Systems, Graduate School of Engineering, Muroran Institute of Technology since 2010. He received the M. Eng. degree and the Ph.D. degree in Engineering from Hokkaido University, Hokkaido, Japan, in 1997 and 2000, respectively. He was a Research Associate in 2003-2005 and an Assistant Professor in 2005-2010 at Department of Computer Science and Systems Engineering, Muroran Institute of Technology, Japan, respectively. He is also a director of Japan Society for Fuzzy Theory and Intelligent Informatics since 2013. He received the Publication Award of Japan Society of Kansei Engineering in 2010. His current research interests are theory and application of rough set, Kansei engineering, non-classical logic, data base, and data mining.

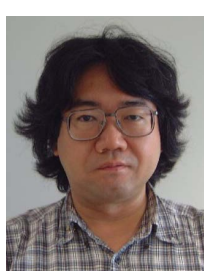

engineering.

\section{Tetsuya MURAI}

Tetsuya Murai is an Associate Professor at Graduate School of Information Science and Technologies, Hokkaido University since 1995 . He received the Dr. Eng degree from Hokkaido University in 1994. His research interests include fuzzy and rough sets, modal logic, granular computing and affective 